\title{
Evaluación del Escarificador MEQT-180X, con tres variedades de quinua (Chenopodium quinoa Willd) para reducir saponina y conservar lisina
}

\author{
Assessment Scarifier MEQT-180X with three varieties of quinoa (Chenopodium quinoa Willd) \\ keep to reduce saponin and lysine \\ Ángela M. Ccora Huamán', Juan F. Ramos Gómez²
}

\section{RESUMEN}

Objetivo: Determinar la eficiencia del equipo escarificador MEQT-180X, en la reducción del contenido de saponina y en la conservación de lisina. Métodos: En el experimento diseñado se utilizó tres factores, variedad de quinua con tres niveles de factor: Pasankalla, Hualhuas y Amarillo Maranganí, velocidad de rotación: 500, 650 y 800 rpm y temperatura del aire de calentamiento: 100 ${ }^{\circ} \mathrm{C}, 150{ }^{\circ} \mathrm{C}$ y $200{ }^{\circ} \mathrm{C}$. Para la conducción del experimento se utilizó un Diseño completamente aleatorio con arreglo factorial de $3 \times 3 \times 3$. Resultados: Las variables respuesta consideradas fueron: contenido de saponina (\%) y contenido de lisina ( $\mathrm{mg} / \mathrm{g}$ de quinua). El menor \% de contenido de saponina se alcanzó utilizando la variedad de quinua pasankalla $0,1504 \pm 0,145$, a una velocidad de rotación del escarificador de $800 \mathrm{rpm}$ y una temperatura de $200^{\circ} \mathrm{C}$. el mayor \% del contenido de saponina se dio en la variedad A. Marangani con el valor de $1,9476 \pm 0,524$. Mientras que el mayor $\%$ del contenido de lisina se conservó utilizando la variedad de quinua Hualhuas, con el valor $54,2368 \pm 2,634$ con velocidad de rotación $500 \mathrm{rpm}$ y $100^{\circ} \mathrm{C}$ de temperatura. Conclusiones: Con el equipo escarificador MEQT-180X se logró determinar el mínimo óptimo del contenido de saponina (\%) en la quinua Pasankalla y la conservación máxima del contenido de lisina ( $\mathrm{mg} / \mathrm{g}$ de quinua) en la variedad de quinua Hualhuas.

Palabras clave: Quinua, quinua escarificada, escarificador, escarificado seco caliente.

\section{ABSTRACT}

Objective: To determine the efficiency of the scaler MEQT-180X team in saponin content reduction and conservation of lysine. Methods: In the experiment designed three factors was used, variety of quinoa with three levels of factor pasankalla, Hualhuas and Yellow Marangani, rotation speed: 500, 650 and $800 \mathrm{rpm}$ and air temperature warming: $100{ }^{\circ} \mathrm{C}, 150{ }^{\circ} \mathrm{C}$ and $200{ }^{\circ} \mathrm{C}$. A A completely randomized design was used with $3 \times 3 \times 3$ factorial arrangement for conducting the experiment was used with $3 \times 3 \times 3$ factorial arrangement for conducting the experiment. Results: The response variables considered were: saponin content (\%) and lysine content (mg / g quinoa). The lowest \% saponin content was achieved using the variety of quinoa pasankalla $0.1504 \pm 0.145$ a rotation speed of $800 \mathrm{rpm}$ scaler and a temperature of $200^{\circ} \mathrm{C}$. the highest \% saponin content was in the range $A$. Marangani with the value of $0.524 \pm 1.9476 \%$ While the highest lysine content was retained using the variety of quinoa Hualhuas, with the value $54.2368 \pm 2,634$ with rotation speed $500 \mathrm{rpm}$ and $100{ }^{\circ} \mathrm{C}$ temperature. Conclusions: With the ripper team MEQT - 180X is able to determine the optimum minimum saponin content (\%) in the pasankalla quinoa and maximum conservation lysine content ( $\mathrm{mg} / \mathrm{g}$ quinoa ) in the range of quinoa Hualhuas .

Keywords: Quinoa, quinoa scarified, scarifier, dry scarification hot. 


\section{INTRODUCCIÓN}

Según Quiroga y Escalera (2010), el episperma es una capa que recubre al grano de quinua. En granos no procesados esta varía desde $20 \mu \mathrm{m}$ en la parte central de las caras hasta más de $100 \mu \mathrm{m}$ en los extremos, cerca al embrión. Esta capa es la que se fragmenta durante el proceso de manipulación de la materia prima, por lo que su remoción es efectiva a través de procesos físicos y químicos. Villacorta y Talavera (1976), mencionan que la saponina se ubica en la primera membrana del epispermo (capa externa). Su contenido y adherencia en los granos es muy variable y ha sido motivo de diferentes estudios y técnicas para eliminarla, por el sabor amargo que confiere al grano. Además del fuerte sabor amargo, se ha descubierto que las saponinas son ligeramente tóxicas para los animales y el ser humano, por ello deben de ser eliminadas antes del consumo del grano (Fortubel y Mirones, 2003).

El perisperma es el principal tejido de almacenamiento y está constituido mayormente por gránulos de almidón, es de color blanquecino y representa prácticamente el $60 \%$ del volumen de la semilla (Armanda, Chavarría y Trejo, 2012).

Las saponinas son glicoalcaloides, factor anti nutricional, que se encuentran en el epispermo de los granos de diversas variedades comerciales de quinua. El contenido de saponinas varía entre $0-3 \%$ en granos secos, aunque se ha reportado variedades con contenidos de saponina de hasta $4 \%$. Granos muy amargos se clasifican entre $1-3 \%$ granos de contenido medio entre 0,1 y $1 \%$ y variedades dulces de 0,0 a 0,15 (Guglú, y Mazza, 2007). Las saponinas están localizadas en el pericarpio de las semillas de la quinua. Dan el sabor amargo y su contenido oscila en el rango de 0,1 al 5,0\% (Pereira, 2011).

Tapia, et al., (1979), mencionan que básicamente se han estudiado hasta el momento cuatro tipos de procesos de desamargado (desaponificación): el seco a temperatura ambiente; el seco en caliente; el húmedo; y el combinado que usa la vía seca y la vía húmeda.

La escarificación, se basa en la fricción entre granos por acción mecánica; para la eliminación de saponina. Generalmente la saponina no es eliminada en su totalidad, sin correr el riesgo de producir rotura de granos, o eliminación de constituyentes importantes como la proteína por desprendimiento de los cotiledones. La escarificación constituye un método muy eficiente para la eliminación de saponinas, pero por solo este medio no es posible la obtención de quinua con niveles suficientemente bajos en saponina para el consumo humano, especialmente si el grano tiene originalmente alto contenido de esta sustancia (Derpic, 1988).

La escarificación no requiere mayor tratamiento previo, sin embargo, su desventaja es que no se logra eliminar completamente toda la saponina del grano; si se pule más al grano, esta operación puede desprender el embrión y hacer que se pierda la proteína que está concentrada en el embrión y la grasa de la capa superior del grano. En este caso el producto obtenido por escarificación alcanzó una eficiencia del 95\% de eliminación, con un contenido final entre 0,04 y $0,25 \%$ de saponina (Torres y Minaya, 1980).

Gaspar, (2014),manifiesta que los dos niveles de temperatura utilizados $\left(17^{\circ} \mathrm{C} \pm 2{ }^{\circ} \mathrm{C}\right.$ y $40^{\circ} \mathrm{C} \pm$ $2{ }^{\circ} \mathrm{C}$ ) en las pruebas experimentales de escarificado, muestran que afectan en forma similar en la eliminación de saponina, habiendo alcanzado contenidos finales de 82,25 y 70,12 $\mathrm{mg}$ saponina/100 g quinua respectivamente, inferior al contenido máximo aceptado por la Norma Técnica Boliviana que es de $120 \mathrm{mg}$ saponina/100 g quinua. Estas mismas dos temperaturas afectaron en forma similar en el contenido final de proteína que fue de $15,29 \%$ y $15,22 \%$ respectivamente, muy cercanos al valor inicial que fue de 15,5\%. Gaspar (2014), también trabajó con velocidades de rotación en el escarificador de 860 y $1146 \mathrm{rpm}$ habiendo encontrado diferencias significativas en el contenido final de saponina $(83,36$ y $69,02 \mathrm{mg}$ saponina/100 g quinua respectivamente), habiendo elegido la velocidad de $1146 \mathrm{rpm}$ como la adecuada. Estas mismas velocidades mostraron afectar en forma similar en el contenido la pérdida de proteína que ocasionó la operación con $1,34 \%$ y $1,83 \%$ respectivamente.

Se han estudiado alternativas tecnológicas para la eliminación de saponina por vía seca (escarificado) en Ecuador. Lo más sobresaliente dentro estas pruebas fue el desarrollo de un modelo de escarificador de quinua de tipo continuo, cuyos resultados 
fueron muy satisfactorios para variedades de bajo contenido de saponina ( $0,2 \%$ o menos), y aceptables para las de alto contenido de saponina. Este prototipo alcanzó a escarificar hasta $75 \mathrm{k} / \mathrm{h}$ de quinua amarga, con $0,026 \%$ de saponina final y $1,5 \%$ de granos quebrados. Con las variedades dulces, se llegó a escarificar hasta $136 \mathrm{k} / \mathrm{h}$, con $0,01 \%$ de saponina final $\mathrm{y}$ $0,0 \%$ de grano quebrado (Valdiviezo y Rivadeneira, 1994).

Según Zavaleta, citado por Tapia (1990), el nivel máximo aceptable de saponina en la quinua para consumo humano oscila entre $0,06 \%$ y $0,12 \%$. Esto concuerda con los resultados de pruebas sensoriales realizados en la Universidad de Ambato, Ecuador en donde se determinó que el límite máximo de aceptación del contenido de saponina en el grano cocido, fue del $0,1 \%$ (Nieto y Soria, 1991).

Soto, Kuramotto, Seleme, Calderón y Castellares, (2010), dan cuenta de los resultados del trabajo del Comité de Normalización de Cereales en Bolivia. La norma NB/NA 038-2007 para granos andinos pseudocereales-Quinua en grano-Clasificación y Requisitos. En lo relacionado al contenido de saponina, presentan el límite máximo de 120 $\mathrm{mg} / 100 \mathrm{~g}$ de quinua determinado por el método espuma.

Mache (2015), Con una velocidad de 900 rpm utilizando un escarificador horizontal de cilindro y lija, con $100 \%$ de abertura del ventilador de succión, logró una remoción del $90,33 \%$ de saponina con pérdida del $4,71 \%$ de proteína en quinua de la variedad Hualhuas. En la variedad Negra Collana logró una remoción del $53,6 \%$ de saponina con pérdida del $4,10 \%$ de proteína a la velocidad de 1390 RPM y ventilador de succión abierto al $50 \%$.

La hipótesis planteada para la investigación, es que un rápido calentamiento seguido de un súbito enfriamiento de la quinua, permitirá la dilatación seguido de una contracción de los granos, lo cual hará más quebradizo al epispermo que los cubre, lo que permitirá aumentar la eficiencia en el desaponificado de la quinua con menores pérdidas de lisina.

El objetivo fue determinar la eficiencia del equipo escarificador MEQT-180X, en la reducción del contenido de saponina y en la conservación de lisina.

\section{MATERIALES Y MÉTODOS}

La materia prima utilizada para la investigación, fue quinua de las variedades: Pasankalla, Hualhuas y Amarillo Marangani, cuya adquisición fue certificada por personal especializado de INIA Huancayo. Entidad Asociada para el desarrollo del proyecto de investigación, con financiamiento de FINCyT, a través del convenio No 191-2012-FINCyTFIDECOM-PIPEA.

El diseño experimental utilizado para la ejecución de la investigación fue el Diseño Completamente Aleatorio con arreglo factorial de $3 \times 3 \times 3$. Los factores de investigación utilizados fueron; Variedad de quinua, con tres niveles de factor: variedad Pasankalla (contenido bajo de saponina), variedad Hualhuas (contenido medio) y variedad Amarillo Marangani (contenido alto). Velocidad de rotación del escarificador, con tres niveles de factor: 500 RPM, 650 RPM y 800 RPM. Finalmente, la Temperatura del aire para el calentamiento de los granos de quinua, con los niveles de factor: $100^{\circ} \mathrm{C}, 150^{\circ} \mathrm{C}$ y $200^{\circ} \mathrm{C}$.

Las variables dependientes consideradas fueron el contenido de Saponina y el contenido de lisina determinados una vez que se aplicaron cada uno de los tratamientos exigidos por el diseño experimental.

Las variables intervinientes fueron: temperatura de enfriamiento de los granos de quinua (hasta temperatura ambiente, entre 17 a $20{ }^{\circ} \mathrm{C}$ ), cantidad de quinua utilizada para cada corrida experimental ( $5 \mathrm{k}$ de quinua), tiempo de escarificación (3,5 min), tiempo de contacto de los granos de quinua con el aire de calentamiento $(1,5$ segundos).El número de repeticiones aplicadas fue de dos, para cada uno de los tratamientos.

El equipo utilizado para realizar las corridas experimentales fue el escarificador diseñado y construido por JARCON DEL PERU SAC, como parte del proyecto convenio No 191-2012FINCyT-FIDECOM-PIPEA, que se muestra en la figura 1.

Este equipo consta de los siguientes sistemas: de calentamiento (1), dosificación de quinua (2), transporte de quinua I (3), colección de producto y descarga (4), transporte de quinua II (5), shock térmico por enfriamiento (6), escarificado (7) y colección de polvos de saponina y residuos (8). 
El calentamiento de aire se realizó para cada tratamiento hasta alcanzar $100^{\circ} \mathrm{C} ; 150^{\circ} \mathrm{C}$ o 200 ${ }^{\circ} \mathrm{C}$, luego se procedió a encender el sistema de aspiración de aire No 2 (transporte de quinua II) y el escarificador (6), para luego abrir la válvula de la tolva de alimentación (2), todos operados desde el panel de control principal. Cada temperatura fue sostenida en forma estable durante toda la corrida experimental haciendo uso de las válvulas para control manual del tamaño de llama en el soplete de calentamiento que son en número de dos). Para la determinación de saponina se utilizó, el método de análisis propuesto por Kosiol (1992), mientras para la determinación de lisina se utilizó el método propuesto por Kadakem
(1969), ambos mencionados por Villegas, Ortega y Bauer (1984).

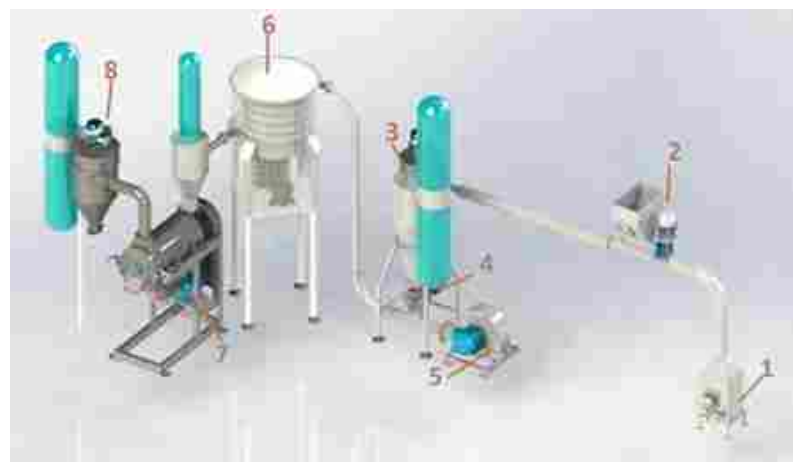

Figura 1. Componentes del Escarificador MEQT-180X

\section{RESULTADOS}

Los datos obtenidos para el contenido de saponina expresado en (\%) al final de cada tratamiento, se muestra en el Tabla 1.

Tabla 1. Contenido de saponina de las muestras de quinua sometido a diferentes tratamientos

\begin{tabular}{|c|c|c|c|c|c|c|c|c|c|}
\hline \multirow{2}{*}{$\begin{array}{l}\text { Temp } \\
\left({ }^{\circ} \mathrm{C}\right)\end{array}$} & \multicolumn{3}{|c|}{ Pasankalla (1) } & \multicolumn{3}{|c|}{ Hualhuas (2) } & \multicolumn{3}{|c|}{ Amarillo Maranganí (3) } \\
\hline & $\begin{array}{c}500 \\
\text { RPM } \\
(1)\end{array}$ & $\begin{array}{c}650 \\
\text { RPM } \\
(2)\end{array}$ & $\begin{array}{c}800 \\
\text { RPM } \\
(3)\end{array}$ & $\begin{array}{c}500 \\
\text { RPM } \\
(1)\end{array}$ & $\begin{array}{c}650 \\
\text { RPM } \\
(2)\end{array}$ & $\begin{array}{c}800 \\
\text { RPM } \\
(3)\end{array}$ & $\begin{array}{c}500 \\
\text { RPM } \\
(1)\end{array}$ & $\begin{array}{c}650 \\
\text { RPM } \\
(2)\end{array}$ & $\begin{array}{c}800 \\
\text { RPM } \\
(3)\end{array}$ \\
\hline \multirow[t]{2}{*}{100 (1) } & 0,231 & 0,231 & 0,231 & 0,231 & 1,259 & 0,032 & 1,48 & 2,656 & 1,995 \\
\hline & 0,054 & 0,157 & 0,525 & 1,554 & 1,113 & 0,032 & 1,186 & 2,877 & 2,068 \\
\hline \multirow[t]{2}{*}{$150(2)$} & 0,032 & 0,054 & 0,341 & 1,186 & 0,377 & 0,745 & 2,656 & 2,582 & 1,554 \\
\hline & 0,032 & 0,098 & 0,377 & 1,039 & 0,098 & 0,231 & 2,215 & 1,995 & 1,884 \\
\hline \multirow[t]{2}{*}{200 (3) } & 0,021 & 0,025 & 0,115 & 0,598 & 0,178 & 0,304 & 1,627 & 2,141 & 1,186 \\
\hline & 0,021 & 0,047 & 0,115 & 0,525 & 0,013 & 0,120 & 2,141 & 1,480 & 1,333 \\
\hline
\end{tabular}

El análisis estadístico de estos datos, muestra que las variedades de quinua Hualhuas, Pasankalla y Amarilla Maranganí, presentan diferencias significativas en el contenido final de saponina, evidenciados en la significación igual a 0,000 . La velocidad de rotación del escarificador en los niveles de factor trabajados (500 RPM, 650 RPM y 800 RPM), afectan significativamente en el contenido final de saponina en los granos de quinua (significación $=0,029)$. La temperatura de calentamiento de los granos de quinua con aire de $100^{\circ} \mathrm{C} ; 150^{\circ} \mathrm{C}$ y $200{ }^{\circ} \mathrm{C}$, también afecta en forma significativa en el contenido final de saponina (Significación $=0,001)$.

La prueba de comparación múltiple de medias (PCMM) de Duncan, para las variedades de quinua, se muestra en la Tabla 2, mientras que para la variable velocidad de rotación los resultados son mostrados en la Tabla 3.

Tabla 2. PCMM de Duncan para los tres niveles del factor variedad de quinua en el contenido final de saponina (\%) en los granos de quinua $\operatorname{con} \alpha=0,05$

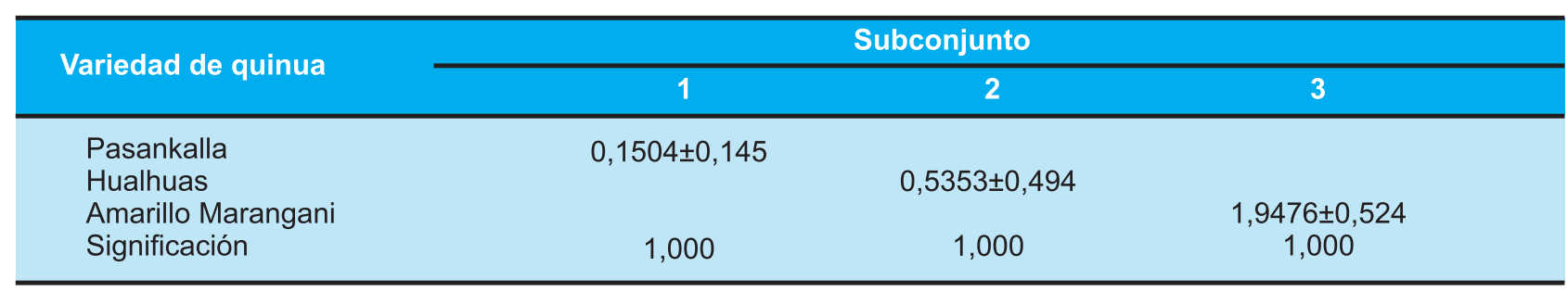


Tabla 3. PCMM de Duncan para los tres niveles del factor velocidad de rotación en el contenido final de saponina (\%) $\operatorname{con} \alpha=0,05$

\begin{tabular}{lcc}
\hline \multirow{2}{*}{$\begin{array}{l}\text { Velocidad } \\
\text { de rotación }\end{array}$} & \multicolumn{2}{c}{ Subconjunto } \\
\cline { 2 - 3 } & 1 & 2 \\
\hline 800 RPM & $0,7327 \pm 0,730$ \\
500 RPM & & $0,9349 \pm 0,864$ \\
650 RPM & & $0,9656 \pm 1,05$ \\
Significación & 1,000 & 0,733 \\
\hline
\end{tabular}

Del análisis de varianza realizado a los datos del contenido de lisina alcanzado por los granos de quinua, luego de la aplicación de cada uno de los tratamientos diseñados, se tiene que el contenido de lisina de las variedades de quinua (Hualhuas, Pasankalla y Amarilla Marangani), presentan diferencias significativas (significación $=0,000$ ). La velocidad de rotación del escarificador en los niveles de factor trabajados (500 rpm, 650 rpm y $800 \mathrm{rpm}$ ), no afectan de forma diferente el
En la Tabla 4 se tiene los resultados de la PCMM de Duncan para la temperatura del aire de calentamiento en el contenido final de saponina.

Tabla 4. PCMM de Duncan para los tres niveles del factor temperatura del aire de calentamiento de los granos de quinua en el Contenido final de saponina (\%) $\operatorname{con} \alpha=0,05$

\begin{tabular}{lcc}
\hline \multirow{2}{*}{ Temperatura } & \multicolumn{2}{c}{ Subconjunto } \\
\cline { 2 - 3 } & \multicolumn{2}{c}{1} \\
\hline $200{ }^{\circ} \mathrm{C}$ & $0,6661 \pm 0,767$ & \\
$150^{\circ} \mathrm{C}$ & & $0,9720 \pm 0,941$ \\
$100^{\circ} \mathrm{C}$ & & $0,9951 \pm 0,943$ \\
significación & 1,000 & 0,797 \\
\hline
\end{tabular}

contenido final de lisina en los granos de quinua (significación $=0,06)$. La temperatura de calentamiento de los granos de quinua con aire de $100{ }^{\circ} \mathrm{C}, 150{ }^{\circ} \mathrm{C}$ y $200{ }^{\circ} \mathrm{C}$ afecta en forma significativa en el contenido final de lisina (Significación $=0,000$ ).

La información del Tabla 5, muestra que los resultados de la PCMM de Duncan de los promedios finales del contenido final de lisina en las tres variedades de quinua.

Tabla 5. PCMM de Duncan para el efecto de los tres niveles del factor variedad de quinua en el contenido final de lisina en los granos de quinua $(\alpha=0,05)$

\begin{tabular}{lccc}
\hline Variedad de quinua & \multicolumn{3}{c}{ Subconjunto } \\
\cline { 2 - 4 } & 1 & 2 & 3 \\
\hline $\begin{array}{l}\text { Pasankalla } \\
\text { Hualhuas }\end{array}$ & $42,2798 \pm 3,048$ & $50,4477 \pm 1,010$ & $54,2368 \pm 2,634$ \\
Amarillo Marangani & & 1,000 & 1,000 \\
\hline
\end{tabular}

En la tabla 6, se tiene los resultados de la PCMM de Duncan del contenido de lisina promedio final de la quinua, cuando estos

granos fueron sometidos a escarificación a velocidades de rotación de 500; 650 y 800 rpm.

Tabla 6. PCMM de Duncan para el Contenido final promedio de lisina en los granos de quinua sometidos a diferentes velocidades de rotación $(\alpha=0,05)$

\begin{tabular}{lcc}
\hline \multirow{2}{*}{ Temperatura } & \multicolumn{2}{c}{ Subconjunto } \\
\cline { 2 - 3 } & 1 & 2 \\
\hline 800 RPM & $48,2749 \pm 5,650$ & \\
650 RPM & $48,8611 \pm 5,462$ & $48,8611 \pm 5,462$ \\
500 RPM & 0,358 & $49,8283 \pm 5,767$ \\
Significación & 0,135 \\
\hline
\end{tabular}

El Tabla 7, muestra los resultados de la PCMM de Duncan, para el contenido de lisina, considerando como variable independiente la temperatura del aire de calentamiento $\left(100^{\circ} \mathrm{C}\right.$, $150^{\circ} \mathrm{C}$ y $200^{\circ} \mathrm{C}$ ). 
Tabla 7. $P C M M$ de Duncan para el Contenido final promedio de lisina ( $\mathrm{mg} / \mathrm{g}$ de proteína) en los granos de quinua calentados con aire $(\alpha=0,05)$

\begin{tabular}{lccc}
\hline Temperatura & \multicolumn{3}{c}{ Subconjunto } \\
\cline { 2 - 4 } & 1 & 2 & 3 \\
\hline $200^{\circ} \mathrm{C}$ & $47,2676 \pm 4,923$ & & \\
$150^{\circ} \mathrm{C}$ & & $48,8669 \pm 6,038$ & $50,8298 \pm 5,373$ \\
$100^{\circ} \mathrm{C}$ & 1,000 & 1,000 & 1,000 \\
Significación & & & \\
\hline
\end{tabular}

Una comparación de los parámetros elegidos que permitió alcanzar los menores contenidos de saponina, comparado con el contenido final de lisina alcanzado por estos, es mostrada en el Tabla 8.

Tabla 8. Contenido de saponina y lisina alcanzado al utilizar los parámetros elegido para generar el menor contenido de saponina.

\begin{tabular}{llcc}
\hline Temperatura & Parámetro & Contenido de Saponina (\%) & $\begin{array}{c}\text { Contenido de } \\
\text { Lisina (mg/g proteína) }\end{array}$ \\
\hline \multirow{3}{*}{ Variedad } & Pasankalla & $0,1504 \pm 0,145$ & $42,2798 \pm 3,048$ \\
& Hualhuas & $0,5353 \pm 0,494$ & $54,2368 \pm 2,634$ \\
Velocidad Rotación & A. Marangani & $1,9476 \pm 0,524$ & $50,4477 \pm 1,010$ \\
& $800 \mathrm{RPM}$ & $0,7327 \pm 0,730$ & $48,2749 \pm 5,650$ \\
Temperatura & $650 \mathrm{RPM}$ & $0,9349 \pm 0,864$ & $48,8611 \pm 5,462$ \\
& $500 \mathrm{RPM}$ & $0,9656 \pm 1,05$ & $49,8283 \pm 5,767$ \\
& $200^{\circ} \mathrm{C}$ & $0,6661 \pm 0,767$ & $47,2676 \pm 4,923$ \\
& $150^{\circ} \mathrm{C}$ & $0,9720 \pm 0,941$ & $48,8669 \pm 6,038$ \\
& $100^{\circ} \mathrm{C}$ & $0,9951 \pm 0,943$ & $50,8298 \pm 5,373$ \\
\hline
\end{tabular}

\section{DISCUSIÓN}

Los datos que se muestran en la tabla 2, evidencian que los tres niveles del factor variedad de quinua afectaron de forma diferente, en el contenido final de saponina, siendo menor en la variedad Pasankalla, seguido de Hualhuas y finalmente amarillo Maranganí, verificándose que en ninguno de tres casos se alcanzó el nivel sugerido por Soto et al. (2010) y por Nieto y Soria (1991), que es del 0,12\%. Sin embargo existe un correlato directo que indica que un mayor contenido inicial de saponina en los granos de quinua, mantiene una cantidad mayor de esta sustancia una vez que fue escarificada.

Los contenidos de saponina alcanzados en las tres variedades se encuentran dentro de lo reportado por Fortubel y Mirones (2003) para quinua sin procesar y de acuerdo a la clasificación propuesta por Pereira (2011), la quinua pasankalla con $0,1504 \%$ de saponina se clasifica como variedad muy dulce, mientras la variedad Hualhuas con $0,5353 \%$ de saponina como variedad de contenido medio, y la variedad Amarillo Maranganí como muy amargo $(1,9474 \%)$.

La información contenida en la Tabla 3, muestra que al utilizar una velocidad de 800 RPM, se alcanza el menor contenido de saponina
$0,7327 \pm 0,730 \%$ significativamente diferente a las otras dos velocidades de rotación (500 y 650 RPM), mientras que al usar las velocidades de 650 y 500 RPM, se alcanzan contenidos de $0,9656 \pm 1,05 \%$ y $0,9349 \pm 0,864 \%$ respectivamente, resultados que no son significativamente diferentes entre sí. Esta información nos permite elegir la velocidad de rotación de 800 RPM como la adecuada para ser usado en el escarificador. Resultado que coincide con lo reportado por Gaspar (2014), que trabajó con velocidades de rotación de 860 y 1146 RPM habiendo encontrado diferencias significativas en el contenido final de saponina para la variedad Hualhuas. Sin embargo el contenido final de $0,7327 \pm 0,730 \%$ de saponina se encuentra muy por encima de lo exigido por la Norma Técnica Boliviana mencionado por Soto, et al (2010), cuyo máximo es del 0,12\% (120 mg saponina/100 g de quinua) y la NTP peruana que obliga que este valor sea cero $(0 \%)$.

El contenido final de saponina, también es afectado por la temperatura del aire de calentamiento. La Tabla 4, indica una relación inversa entre la temperatura de calentamiento y el contenido final de saponina, es así que al usar aire de $100^{\circ} \mathrm{C}$ el contenido final de saponina es de $0,9951 \pm 0,943 \%$ y cuando se utiliza aire de $150{ }^{\circ} \mathrm{C}$ alcanza el $0,9720 \pm 0,941 \%$ de saponina (no existiendo diferencia significativa en el efecto de 
estas dos temperaturas), mientras que cuando se utiliza aire de $200{ }^{\circ} \mathrm{C}$ el contenido final de saponina es de 0,6661 $0,767 \%$, significativamente diferente y menor al alcanzado con las dos temperaturas anteriores $\left(100{ }^{\circ} \mathrm{C}\right.$ y $\left.150^{\circ} \mathrm{C}\right)$, Esto indica que la temperatura del aire de $200^{\circ} \mathrm{C}$, es la mejor para ser utilizada en el trabajo de escarificación en el equipo diseñado y construido, resultado diferente al obtenido por Gaspar (2014), que demostró que la diferentes temperaturas de calentamiento de los granos de quinua de la variedad Hualhuas antes del escarificado afectaron en forma similar en la eliminación de saponina, habiendo utilizado las temperaturas de $17^{\circ} \mathrm{C} \pm 2{ }^{\circ} \mathrm{C}$ y $40^{\circ} \mathrm{C} \pm 2{ }^{\circ} \mathrm{C}$. En la investigación el efecto de la temperatura del aire es significativo, porque estas son muy superiores a las utilizadas por Gaspar, (2014). Igual que en los casos anteriores ninguna de las muestras se aproxima al contenido de saponina exigido por las Norma Técnica Boliviana $(0,12 \%$ o 120 mg saponina/100 g de quinua).

La información de la Tabla 5, muestra que la variedad Hualhuas es la que presenta el mayor contenido de lisina luego de aplicado los tratamientos correspondientes $54,2368 \pm 2,634$ $\mathrm{mg}$ lisina/g de proteína, seguido del Amarillo Marangani con 50,4477 $\pm 1,010$; luego la variedad Pasankalla con $42,2798 \pm 3,048$. Con esta información se determina que la variedad de quinua que conserva mayor contenido de lisina después de la escarificación es Hualhuas. Lo que coincide con lo demostrado por Gaspar (2014), que determinó que la variedad Hualhuas solo pierde entre $1,43 \%$ y $1,83 \%$ de proteína presente en el grano cuando es escarificado, que se ve traducido en una mejor conservación de este aminoácido esencial contenido como parte de la proteína.

La Tabla 6, muestra, que no existen diferencias significativas en el contenido final de lisina, entre los granos de quinua escarificados a $800 ; 650$ RPM, y tampoco existen diferencias significativas cuando se compara los niveles de factor de $500 \mathrm{y}$ 650 RPM. Siendo finalmente la diferencia entre una y otra muy pequeña, por lo que cualquiera de las tres velocidades de rotación puede ser elegida con iguales beneficios. Este resultado es coincidente con lo reportado por Gaspar (2014), que no encontró diferencias significativas en el contenido final de proteína cuando escarificó quinua variedad Hualhuas a las velocidades de 860 y $1146 \mathrm{rpm}$.

LaTabla 7, muestra que la temperatura del aire de calentamiento que mejor conserva el contenido final de lisina, es el de $100^{\circ} \mathrm{C}, 50,8298 \pm 5,373 \mathrm{mg}$ lisina/g de proteína, mientras al utilizar aire de 150 ${ }^{\circ} \mathrm{C}$ se alcanza 48,8669 $\pm 6,038$ y al usar aire de 200 ${ }^{\circ} \mathrm{C}$ la cantidad de lisina que aún conserva el grano de quinua es de $47,2676 \pm 4,923$. Esto indica que la temperatura del aire de $100^{\circ} \mathrm{C}$, es la que mejor conserva la lisina en los granos de quinua. Este resultado difiere bastante de lo reportado por Gaspar (2014), quien no encontró diferencias significativas en el contenido final de proteína cuando utilizó temperaturas de calentamiento de los granos de quinua de $17^{\circ} \mathrm{C} \pm 2{ }^{\circ} \mathrm{C}$ y $40^{\circ} \mathrm{C} \pm 2$ ${ }^{\circ} \mathrm{C}$, seguramente porque estas temperaturas son bastante bajas en comparación a las temperaturas utilizadas en la presente investigación $\left(100{ }^{\circ} \mathrm{C}, 150{ }^{\circ} \mathrm{C}\right.$ y $\left.200{ }^{\circ} \mathrm{C}\right)$. De la Tabla 7 , también se desprende que una mayor temperatura del aire de calentamiento afecta en mayor medida en la desnaturalización de la lisina, general un menor contenido de este al final de la operación de escarificación.

Una comparación de los parámetros elegidos que permiten alcanzar los menores contenidos de saponina, comparado con el contenido final de lisina alcanzado por estos, es mostrada en la Tabla 8. Aquí puede verificarse claramente, que la variedad Pasankalla que alcanza el que menor contenido de saponina $0,1504 \%$, es el que menos lisina conserva luego de escarificado (solo $42,2798 \mathrm{mg}$ lisina/g proteína). Este mismo comportamiento se tiene en relación a la velocidad de escarificación donde con 800 rpm se logró el menor contenido de saponina $(0,5353 \%)$, pero también el menor contenido de lisina $(48,2749 \mathrm{mg} / \mathrm{g}$ quinua). Lo mismo se da en cuanto a la temperatura del aire de calentamiento de los granos de quinua, donde con $200^{\circ} \mathrm{C}$ se alcanza el menor contenido de saponina $(0,6661 \%)$, pero también es el que más lisina sacrifica en la operación (solo conserva 47,2676 $\mathrm{mg}$ lisina/g proteína.

\section{AGRADECIMIENTOS}

Agradecimientos especiales al Fondo de Investigación y Desarrollo para la Competitividad FIDECOM del Perú, que apostó por la ejecución del proyecto, a través del convenio No 191-2012FINCYT-FIDECOM-PIPEA.

\section{REFERENCIAS BIBLIOGRÁFICAS}

Armanda, M. Chavarría, J. \& Trejo A. (2012). Diseño y Construcción de un Equipo Escarificador de Quinua. Revista Argentina de Ingeniería, 1, 53-58. 
Derpic, E. (1988). Sistema Combinado para la Desaponificación de Quinua. Memorias del VI Congreso Internacional Sobre Cultivos Andinos. Quito.

Fortubel, F. \& Mirones, G. (2003). Los Tractores del "Mallku" Convertirán el Altiplano en Desierto. Semanario La Época. p. B3.

Gaspar, D. (2014). Estudio de los factores que afectan en el desamargado de quinua (Chenopodium quinoa Willd) por vía seca utilizando un pulidor de cebada. Tesis para optar el grado de Ingeniero en Industrias Alimentarias. Huancayo: Universidad Nacional del Centro del Perú.

Guglú, O. \& Mazza, G. (2007). Saponins: Properties, Applications and Processing. Critical Reviews in Food Science and Nutrition. 47, 231-258.

Koziol M.J. (1992). Chemical Composition and nutritional evaluation of quinoa (Chenopodium quinua Willd). J. Food Comp. Anal., 5, 35-68.

Mache, J. (2015). Efecto de la velocidad de giro, nivel de abertura del escarificador, velocidad de agitación y tiempo de lavado en el contenido final de saponina y proteínas de la quinua (Chenopodium quinoa Willd). Tesis para optar el grado de Ingeniero en Industrias Alimentarias. Huancayo: Universidad Nacional del Centro del Perú.

Nieto, C. \& Soria, M. (1991). Procesamiento de quinua en Ecuador, Proyecto 3P-850213. Informe Final de Labores. Quito: INIAP-UTA-CIID.

Pereira, S. (2011). Elaboración de Leche de Quinua. Proyecto Previo para optar el grado de Ingeniero Agroindustrial. Quito: Escuela Politécnica Nacional.

Quiroga, C. \& Escalera, R. (2010). Evaluación de la Calidad Nutricional y Morfología del Grano de Variedades Amargas de Quinua Beneficiadas en Seco, Mediante el Empleo del Novedoso Empleo de un Reactor de Lecho Fluidizado de Tipo Surtidor. Revista Investigación y Desarrollo. 10, 23-36.

Soto, J., Kuramotto, C., Seleme, J., Calderón, R. \& Castellares, C. (2010). Normas
Andinas para quinua (Chenopodium quinoa Willd) y productos procesados (hojuelas y harina). Comité Técnico 3.12 Cereales Quinua IBNORCANOREXPORT. Bolivia.

Tapia, M., Gandarillas, H., Aladia, S., Cardozo, A., Mujica, A., Ortiz, R., Otaju, V., Rea, J., Salas, B. y zanabria, E. (1979). La Quinua y la Kañiwa. Centro Internacional de Investigaciones para el Desarrollo, Instituto Internacional de Ciencias Agrícolas, Bogotá, Colombia.

Tapia, M. (1990). Cultivos Andinos subexplotados y su aporte a la alimentación. Santiago. FAO. Ediciones Gegra S.A.

Torres, H. \& Minaya, I. (1980). Escarificadora de Quinua, diseño y construcción. Instituto Interamericano de Ciencias Agrícolas. Publicaciones misceláneas $\mathrm{N}^{\circ} 243$, Lima, Perú 1980. 27 p

Valdiviezo, J. \& Rivadeneira, V. (1994). Diseño y construcción de una escarificadora de quinua por vía seca en un flujo continuo. Tesis para la obtención del Título de Ingeniero Mecánico. Quito: Escuela Politécnica Nacional.

Villacorta, S. \& Talavera, V. (1976). Anatomía del Grano de Quinua (Chenopodium quinoa Willd). Revista Anales Científicos UNA, 14:39-45.

Villegas, E., Ortega, E y Bauer, R. (1984). Chemical methods used at CIMMYT for determining protein quality in cereal grains. CIMMYT. Mexico, D. F. 35 p

Correo electrónico: jragouncp@yahoo.es

Revisión de Pares:

Recibido: 01-10-2015

Aceptado: 21-12-2015 